\title{
ThermoData Engine (TDE): Software Implementation of the Dynamic Data Evaluation Concept ${ }^{1}$ (Supporting Information)
}

\author{
Michael Frenkel, ${ }^{2}$ Robert D. Chirico, Vladimir Diky, Xinjian Yan, ${ }^{3}$ Qian Dong, \\ and Chris Muzny
}

Physical and Chemical Properties Division

National Institute of Standards and Technology (NIST)

Boulder, CO 80305-3328, U.S.A.

${ }^{1}$ Contribution of the National Institute of Standards and Technology not subject to copyright in the United States.

2 To whom correspondence should be addressed. Email: frenkel@boulder.nist.gov

${ }^{3}$ Current Address: Scientific Information Center, Thornton, Colorado 80241. E-mail: yunyan316@yahoo.com. 
Equations for property representations in TDE are listed here. Units for all properties and variables are given in the following table.

\begin{tabular}{|l|c|l|}
\hline Name & Symbol Unit \\
\hline temperature & $T$ & $\mathrm{~K}$ \\
\hline pressure & $p$ & $\mathrm{kPa}$ \\
\hline density & $\rho$ & $\mathrm{kg} \cdot \mathrm{m}^{-3}$ \\
\hline enthalpy of vaporization & $\Delta_{\mathrm{vap}} H$ & $\mathrm{~kJ} \cdot \mathrm{mol}$ \\
\hline heat capacity at saturation pressure & $C_{\mathrm{sat}}$ & $\mathrm{J} \cdot \mathrm{K}^{-1} \mathrm{~mol}^{-1}$ \\
\hline heat capacity at constant pressure & $C_{\mathrm{p}}$ & $\mathrm{J} \cdot \mathrm{K}^{-1} \mathrm{~mol}^{-1}$ \\
\hline speed of sound & $v$ & $\mathrm{~m} \cdot \mathrm{s}^{-1}$ \\
\hline refractive index (Na D-line) & $n_{\mathrm{D}}$ & $\mathrm{dimensionless}$ \\
\hline surface tension & $\sigma$ & $\mathrm{N} \cdot \mathrm{m}^{-1}$ \\
\hline viscosity & $\eta$ & $\mathrm{Pa} \cdot \mathrm{s}$ \\
\hline thermal conductivity & $\lambda$ & $\mathrm{W} \cdot \mathrm{m}^{-1} \cdot \mathrm{K}^{-1}$ \\
\hline
\end{tabular}

\section{Vapor Pressure p: Liquid-Gas Phase Boundary Pressure}

- DEFAULT: Wagner 25

$\ln \left(p / p^{0}\right)-\ln \left(p / p^{9}\right)=T_{d} / T\left(\mathrm{~A}_{1}+\mathrm{A}_{2} \cdot \tau^{1.5}+\mathrm{A}_{3} \cdot \tau^{2.5}+\mathrm{A}_{4} \cdot \tau^{5}\right) ;$ where $\tau=1$ $T / T_{c}$ and $p^{\circ}=1 \mathrm{kPa}$

- DEFAULT: PV Expansion: PVExpansion $\ln \left(p / p^{9}\right)=a_{1}+a_{2} / T+a_{3} \cdot \ln (T)+a_{4} \cdot T+a_{5} \cdot T^{2}+a_{6} / T^{2}+a_{7} \cdot T^{6}+a_{8} / T^{4}$

- Alternative 1: Antoine $\ln \left(p / p^{o}\right)=\mathrm{A}+\mathrm{B} /(\mathrm{T}+\mathrm{C}) ;$ where $p^{o}=1 \mathrm{kPa}$

- Alternative 2: Yaws.VaporPressureYaws-VaporPressure $\lg \left(p / p^{0}\right)=\mathrm{a}+\mathrm{b} / \mathrm{T}+\mathrm{c} \lg (\mathrm{T})+\mathrm{d} \cdot \mathrm{T}+\mathrm{e} / \mathrm{T}^{2} ;$ where $p^{\circ}=1 \mathrm{kPa}$ and $\lg =$ $\log _{10}$

- Alternative 3: DIPPR 115 DIPPR115 $\ln \left(p / p^{0}\right)=\mathrm{a}+\mathrm{b} / \mathrm{T}+\mathrm{c} \cdot \ln (\mathrm{T})+\mathrm{d} \cdot \mathrm{T}^{2}+\mathrm{e} / \mathrm{T}^{2} ;$ where $p^{\circ}=1 \mathrm{kPa}$

- Alternative 4: DIPPR 101 DIPPR101 $\ln \left(p / p^{\circ}\right)=\mathrm{a}+\mathrm{b} / \mathrm{T}+\mathrm{c} \cdot \ln (\mathrm{T})+\mathrm{d} \cdot \mathrm{T}^{\mathrm{e}} ;$ where $p^{\circ}=1 \mathrm{kPa}$

- Alternative 5: Wagner 36 Wagner36

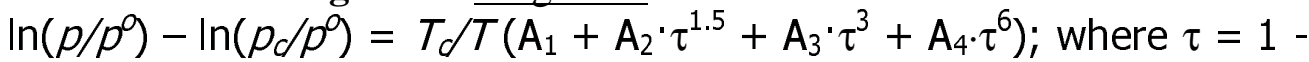
$T / T_{c}$ and $p^{\circ}=1 \mathrm{kPa}$

Sublimation Pressure p: Crystal-Gas Phase Boundary Pressure

- DEFAULT: PV Expansion: PVExpansion $\ln \left(p / p^{9}\right)=a_{1}+a_{2} / T+a_{3} \cdot \ln (T)+a_{4} \cdot T+a_{5} \cdot T^{2}+a_{6} / T^{2}+a_{7} \cdot T^{6}+a_{8} / T^{4}$

- Alternative 1: Antoine Antoine $\ln \left(p / p^{\circ}\right)=\mathrm{A}+\mathrm{B} /(\mathrm{T}+\mathrm{C})$; where $p^{\circ}=1 \mathrm{kPa}$

- Alternative 2: Yaws.VaporPressure Yaws-VaporPressure 
$\lg \left(p / p^{0}\right)=\mathrm{a}+\mathrm{b} / \mathrm{T}+\mathrm{c} \cdot \lg (\mathrm{T})+\mathrm{d} \cdot \mathrm{T}+\mathrm{e} / \mathrm{T}^{2} ;$ where $p^{\circ}=1 \mathrm{kPa}$ and $\lg =$ $\log _{10}$

- Alternative 3: DIPPR 115 DIPPR115 $\ln \left(p / p^{\circ}\right)=\mathrm{a}+\mathrm{b} / \mathrm{T}+\mathrm{c} \cdot \ln (\mathrm{T})+\mathrm{d} \cdot \mathrm{T}^{2}+\mathrm{e} / \mathrm{T}^{2} ;$ where $p^{\circ}=1 \mathrm{kPa}$

- Alternative 4: DIPPR 101 DIPPR101 $\ln \left(p / p^{\circ}\right)=\mathrm{a}+\mathrm{b} / \mathrm{T}+\mathrm{c} \cdot \ln (\mathrm{T})+\mathrm{d} \cdot \mathrm{T}^{\mathrm{e}} ;$ where $p^{\rho}=1 \mathrm{kPa}$

Condensed Phase boundary pressure $p$

- DEFAULT: PolynomialPressure: PolynomialPressureCL $\ln \left(p / p^{0}\right)=\sum \mathrm{a}_{\mathrm{i}} \cdot T^{\mathrm{i}}$, where the summation is from $\boldsymbol{i}=\boldsymbol{0}$ to $\boldsymbol{n T e r m s}-\boldsymbol{1}$

Density of the Liquid $\rho$ (on the Liquid/Gas phase boundary): $\rho_{\text {sat }}$

- DEFAULT: VDNS Expansion: VDNSExpansion $\rho_{\text {sat }}=\rho_{c}+\mathrm{a}_{1} \cdot \tau^{0.35}+\sum \mathrm{a}_{\mathrm{i}+1} \cdot \tau^{i}$, where the summation is from $\boldsymbol{i}=\mathbf{1}$ to nTerms $\mathbf{- 1}$. $\rho_{c}=$ the critical density and $\tau=1-T / T_{c}$, where $T_{c}$ is the critical temperature.

- Alternative 1: Rackett Rackett $\rho_{\text {sat }}=\rho_{c} \cdot \mathrm{B}^{-(1-T / T) \mathrm{N}}$; where $T_{c}$ and $\rho_{c}$ are the critical temperature and critical density, respectively.

- Alternative 2: PPDS 10 PPDS10 $\rho_{\text {sat }}=d_{c}+\mathrm{a}_{1} \cdot \tau^{0.35}+\mathrm{a}_{2} \cdot \tau^{2 / 3}+\mathrm{a}_{3} \cdot \tau+\mathrm{a}_{4} \cdot \tau^{4 / 3}$ $\rho_{c}=$ the critical density and $\tau=1-T / T_{c}$, where $T_{c}$ is the critical temperature.

- Alternative 3: PPDS 17 PPDS17 $\rho_{\text {sat }}=\left(a_{1}+a_{2} \cdot \tau\right)^{-1-\tau^{2 / 7}} / a_{0} ;$ where $\tau=1-T / T_{c}$ and $T_{c}$ is the critical temperature.

Density of the Gas $\rho$ (on the Liquid/Gas phase boundary)

- DEFAULT: VDNSExpansion: VDNSExpansion $\rho_{\text {sat }}=d_{c}+\mathrm{a}_{1} \cdot \tau^{0.35}+\sum \mathrm{a}_{\mathrm{i}+1} \cdot \tau^{i}$, where the summation is from $\boldsymbol{i}=\mathbf{1}$ to nTerms $\mathbf{- 1}$.

$\rho_{c}=$ the critical density and $\tau=1-T / T_{c}$ where $T_{c}$ is the critical temperature.

Density $\rho$ (Liquid Phase)

- DEFAULT: Tait Equation: TaitEquation

$\rho=\rho_{\text {sat }} /\left[1-\mathrm{c} \cdot \ln \left\{(\mathrm{b}+p) /\left(\mathrm{b}+p_{\text {sat }}\right)\right\}\right]$; where

$\mathrm{b}=\Sigma \mathrm{B}_{i+1} \cdot \tau^{i}, \mathrm{c}=\Sigma \mathrm{C}_{i+1} \cdot \tau^{i}$, and $\tau=\left(T-T_{\text {center }}\right) / 100$, where $T_{\text {center }}$ is a constant parameter.

Summations are from $\mathrm{i}=0$ to (nTerms -1$)$, where $n$ Terms is the number of $\mathrm{B}$ or $\mathrm{C}$ terms.

$\rho_{\text {sat }}=$ the density of the saturated liquid and $p_{\text {sat }}=$ the saturation vapor pressure at temperature $T$.

Density $d$ (Gas Phase)

- DEFAULT: Virial Equation (VirialV): VirialV-GasDensity 
$\rho=1000 \cdot \mathrm{Mw} / V_{m} ;$ where $\mathrm{Mw}$ is the molar mass (in $\mathrm{kg} / \mathrm{kmol}$ ) and $V_{m}$ is the molar volume.

$\mathrm{Z}=1000 \cdot p \cdot V_{m} /(\mathrm{R} \cdot T)=1+\mathrm{B} / V_{m}+\mathrm{C} / V_{m}^{2}$,

where $\mathrm{B}=\sum \mathrm{b}_{i+1} / T^{i}$ and $\mathrm{C}=\sum \mathrm{c}_{\mathrm{i}+1} / T^{\mathrm{i}}$; summation fro $\mathrm{i}=0$ to $n$ Terms 1 ;

$p$ is pressure $(\mathrm{kPa})$.

\section{Enthalpy of Vaporization $\Delta_{\mathrm{vap}} H$ (Liquid/Gas)}

- DEFAULT: HVP Expansion: HVPExpansion

$\ln \left(\Delta_{\text {vap }} H / \Delta_{\text {vap }} H^{P}\right)=\mathrm{a}_{1}+\sum \mathrm{a}_{\mathrm{i}} \cdot T_{r}^{\mathrm{i}-2} \cdot \ln \left(1-T_{r}\right)$, where the summation is from $\boldsymbol{i}$ $=2$ to $n$ Terms -1

$T_{r}=T / T_{c}, T_{c}$ is the critical temperature, and $H_{v a p}{ }^{0}=1 \mathrm{~kJ} / \mathrm{mol}$

- Alternative 1: Yaws. VaporizationH Yaws. VaporizationH

$\Delta_{\mathrm{vap}} H=\mathrm{A} \cdot\left\{1-\left(T / T_{C}\right)\right\}^{n}$

- Alternative 2: PPDS 12 PPDS12

$\Delta_{\text {vap }} H /\left(R \cdot T_{c}\right)=a_{1} \cdot \tau^{1 / 3}+a_{2} \cdot \tau^{2 / 3}+a_{3} \cdot \tau+a_{4} \cdot \tau^{2}+a_{5} \cdot \tau^{6}$

where $\tau=1-T / T_{c}, T_{c}$ is the critical temperature, and $\mathrm{R}$ is the gas

constant.

Heat capacity at vapor saturation $C_{\text {sat }}$ (Liquid Phase)

- DEFAULT: CSExpansion: CSExpansion

$C_{\text {sat }}=\left(\sum \mathrm{a}_{i+1} \cdot \tau^{\mathrm{i}}\right)+\mathrm{b} / \tau$, where the summation is from $\boldsymbol{i}=\mathbf{0}$ to $\boldsymbol{n T e r m s} \mathbf{- 1}$.

- Alternative 2: Yaws.PolynomialExpansion Yaws.PolynomialExpansionCsat $C_{s a t}=\sum a_{i} \cdot T^{i}$, where the summation is from $\boldsymbol{i}=\mathbf{0}$ to $\boldsymbol{n T e r m s}-\boldsymbol{1}$

- Alternative 3: DIPPR.PolynomialExpansion DIPPR.PolynomialExpansionCsat $C_{\text {sat }}=\sum \mathrm{a}_{\mathrm{i}} \cdot T^{\mathrm{i}}$, where the summation is from $\boldsymbol{i}=\mathbf{0}$ to $\boldsymbol{n T e r m \boldsymbol { s }}-\boldsymbol{1}$

- Alternative 3: PPDS 15 PPDS15-Csat

$C_{\text {sat }} / \mathrm{R}=\mathrm{a}_{0} / \tau+\mathrm{a}_{1}+\mathrm{a}_{2} \cdot \tau+\mathrm{a}_{3} \cdot \tau^{2}+\mathrm{a}_{4} \cdot \tau^{3}+\mathrm{a}_{5} \cdot \tau^{4}$

where $\tau=1-T / T_{c}, T_{c}$ is the critical temperature, and $\mathrm{R}$ is the gas constant.

Heat Capacity $C_{p}{ }^{\circ}$ (Ideal Gas)

- DEFAULT: Wilhoit Equation

$C_{p}^{0} / \mathrm{R}=a_{0}+\left(a_{1} / T^{2}\right) \cdot \exp \left(-a_{2} / T\right)+a_{3} \cdot y^{2}+\left\{a_{4}-a_{5} /\left(T-a_{7}\right)^{2}\right\} \cdot y^{8}$ where $y=\left(T-a_{7}\right) /\left(T+a_{6}\right)$ and $\mathrm{R}$ is the gas constant.

See Thermodynamics of Organic Compounds in the Gas State (Volumes I and II) by M. Frenkel, G. J. Kabo, K. N. Marsh, G. N. Roganov, and R. C. Wilhoit. TRC, College Station: TX. 1994.

- Alternative 1: Yaws.PolynomialExpansion Yaws.PolynomialExpansionCp0 $C_{p}{ }^{0}=\sum \mathrm{a}_{\mathrm{i}}{ }^{\mathrm{i}}$, where the summation is from $\boldsymbol{i}=\mathbf{0}$ to $\boldsymbol{n T e r m s}-\boldsymbol{1}$

- Alternative 2: AlyLee (which is also DIPPR 107) $\mathrm{AlyLeeCp0}$ $C_{p}^{0}=\mathrm{a}+\mathrm{b} \cdot\{(\mathrm{c} / \mathrm{T}) / \sinh (\mathrm{c} / \mathrm{T})\}^{2}+\mathrm{d} \cdot\{(\mathrm{e} / \mathrm{T}) / \cosh (\mathrm{e} / \mathrm{T})\}^{2}$

- Alternative 3: PPDS2 PPDS2Cp0

$C_{p}{ }^{o} / \mathrm{R}=\mathrm{C}_{\text {low }}+\left(\mathrm{C}_{\text {low }}-\mathrm{C}_{\infty}\right)^{\cdot} \mathrm{y}^{2} \cdot\left\{1+(\mathrm{y}-1) \sum\left(\mathrm{a}_{\mathrm{i}}{ }^{\mathrm{y}} \mathrm{y}_{\mathrm{i}}\right)\right\}$; where the summation is from $i=0$ to 4 .

$\mathrm{C}_{\text {low }}$ and $\mathrm{C}_{\infty}$ are equation constants, and $\mathrm{y}=T /\left(T+\mathrm{T}_{\mathrm{S}}\right)$, where $\mathrm{T}_{\mathrm{S}}$ is a constant.

Speed of Sound $v$ (Liquid Phase) 
- DEFAULT: RSSL Expansion: RSSL-Expansion

$v=\mathrm{A}+\mathrm{B} \cdot \rho_{\text {sat }}+\mathrm{C} \cdot\left(\rho-\rho_{\text {sat }}\right)+\mathrm{D} \cdot \rho^{2}$,

where $\rho$ is the density of the liquid and $\rho_{\text {sat }}$ is the density of the saturated liquid.

Speed of Sound v (Gas Phase)

- DEFAULT: RSSG Expansion: RSSG-Expansion

$v=\mathrm{A}+\mathrm{B} \cdot T+\mathrm{C} \cdot p+\mathrm{D} \cdot p / T$;

where $T$ is the temperature and $p$ is pressure.

Refractive Index $n_{\mathrm{D}}$ (Liquid)

- DEFAULT: RIXExpansion: RIXExpansion

$n_{\mathrm{D}}=\mathrm{A}+\mathrm{B} \cdot t+\sum \mathrm{C}_{\mathrm{i}} \cdot \boldsymbol{w}$; the summation is from $\boldsymbol{i}=\mathbf{1}$ to $\boldsymbol{n T e r m s}$

where $t=T-298.15 \mathrm{~K}$ and $w=W L-589.26$ ( $W L=$ wavelength in $\mathrm{nm})$

Surface Tension $\sigma$ (Liquid/Gas)

- DEFAULT: ISTExpansion: PPDS14

$\sigma=\sum a_{i}{ }^{i} \tau^{i}$, where $\tau=1-T / T_{c}$ and $T_{c}$ is the critical temperature.

Summation is from $i=1$ to $n$ Terms.

- DEFAULT: PPDS 14: PPDS14

$\sigma=a_{0} \cdot \tau_{1}^{a} \cdot\left(1+a_{2} \cdot \tau\right)$, where $\tau=1-T / T_{c}$ and $T_{c}$ is the critical

temperature.

- Alternative 1: Yaws.SurfaceTension Yaws.SurfaceTension $\sigma=\exp (\mathrm{A}) \cdot\left\{1-\left(T / T_{c}\right)\right\}^{n}$

- Alternative 2: HVPExpansion HVPExpansion-SurfaceTension $\ln \left(\sigma / \sigma^{0}\right)=\mathrm{a}_{1}+\sum \mathrm{a}_{\mathrm{i}} \cdot T_{r}^{\mathrm{i}-2} \cdot \ln \left(1-\overline{T_{r}}\right)$, where the summation is from $\boldsymbol{i}=\mathbf{2}$ to nTerms -1

$T_{r}=T / T_{c}, T_{c}$ is the critical temperature, and $\sigma^{0}=1 \mathrm{~N} / \mathrm{m}$

Viscosity $\eta$ (Saturated Liquid)

- DEFAULT: ViscosityL: PPDS9-ViscosityLiquid/Gas

$\ln \left(\eta / \eta^{\circ}\right)=\mathrm{a}_{0}+\mathrm{a}_{1} / \mathrm{T}+\mathrm{a}_{2} / \mathrm{T}^{2}+\mathrm{a}_{3} / \mathrm{T}^{3}, \eta^{0}=1 \mathrm{~Pa} \cdot \mathrm{s}$

- DEFAULT: PPDS 9: PPDS9-ViscosityLiquid/Gas

$\ln \left(\eta / \eta^{0}\right)=\mathrm{a}_{1} \cdot \mathrm{X}^{1 / 3}+\mathrm{a}_{2} \cdot \mathrm{X}^{4 / 3}+\ln \left(\mathrm{a}_{5}\right)$, where $\mathrm{X}=\left(\mathrm{a}_{3}-T\right) /\left(T-\mathrm{a}_{4}\right)$ and $\eta^{0}$ $=1 \mathrm{~Pa} \cdot \mathrm{S}$

- Alternative 1: Yaws.Viscosity YawsViscosity $\lg \left(\eta / \eta^{\circ}\right)=\mathrm{A}+\mathrm{B} / T+\mathrm{C} \cdot T+\mathrm{D} \cdot T^{2}$

where $\lg =\log _{10}$.

- Alternative 2: DIPPR 101 DIPPR101-Viscosity $\ln \left(\eta / \eta^{0}\right)=\mathrm{a}+\mathrm{b} / \mathrm{T}+\mathrm{c} \cdot \ln (\mathrm{T})+\mathrm{d} \cdot \mathrm{T}^{\mathrm{e}} ;$ where $\eta^{0}=1 \mathrm{~Pa} \cdot \mathrm{S}$

Viscosity $\eta$ (Gas at low pressures; $p<6$ bar)

- DEFAULT: TransportPolynomial: TransportPolynomial-ViscosityGas $\eta=\sum \mathrm{a}_{\mathrm{i}} \cdot T^{\mathrm{i}}$, where the summation is from $\boldsymbol{i}=\mathbf{0}$ to $\boldsymbol{n T e r m s}-\boldsymbol{1}$

- Alternative 1: Yaws.PolynomialExpansion Yaws.PolynomialExpansionViscosityGas

$\eta=\sum a_{i} \cdot T^{i}$, where the summation is from $\boldsymbol{i}=\boldsymbol{O}$ to $\boldsymbol{n T e r m \boldsymbol { s }}-\boldsymbol{1}$

- Alternative 2: DIPPR 102 DIPPR102-vISCOSITYgAS 


$$
\eta=\mathrm{A} \cdot T^{\mathrm{B}} /\left(1+\mathrm{C} / T+\mathrm{D} / T^{2}\right)
$$

- Alternative 3: PPDS 5 PPDS5-ViscosityGas

$\eta=\mathrm{a}_{0} \cdot T_{r} /\left\{1+\mathrm{a}_{1} \cdot\left(T_{r}-1\right) \cdot \mathrm{T}_{\mathrm{r}}{ }_{2}\right\}^{1 / 6} ;$ where $T_{r}=T / T_{c}$ and $T_{c}$ is the critical temperature.

Thermal Conductivity $\lambda$ : (Liquid)

- DEFAULT: TransportPolynomial: TransportPolynomial $\lambda=\sum \mathrm{a}_{\mathrm{i}} \cdot \boldsymbol{T}^{\mathrm{i}}$, where the summation is from $\boldsymbol{i}=\mathbf{0}$ to $\boldsymbol{n T e r m \boldsymbol { s }} \mathbf{- 1}$

- Alternative 1: Yaws.ThermalConductivity Yaws.ThermalConductivity $\lg \left(\lambda / \lambda^{0}\right)=A+B^{\cdot}(1-7 / C)^{2 / 7}$

- Alternative 2: Yaws.PolynomialExpansion Yaws.PolynomialExpansion $\lambda=\sum \mathrm{a}_{\mathrm{i}}{ }^{*} \boldsymbol{T}^{\mathrm{i}}$, where the summation is from $\boldsymbol{i}=\boldsymbol{0}$ to $\boldsymbol{n T e r m s}-\boldsymbol{1}$

- Alternative 3: DIPPR.PolynomialExpansion DIPPR.PolynomialExpansion $\lambda=\sum \mathrm{a}_{\mathrm{i}} \cdot T^{\mathrm{i}}$, where the summation is from $\boldsymbol{i}=\boldsymbol{0}$ to $\boldsymbol{n T e r m \boldsymbol { s }} \boldsymbol{- 1}$

- Alternative 4: PPDS 8 PPDS8 $\lambda=\mathrm{a}_{1}+\sum\left(\mathrm{a}_{\mathrm{i}+1} \cdot \tau^{\mathrm{i} / 3}\right)$, where the summation is from $\boldsymbol{i}=\mathbf{1}$ to $\boldsymbol{n T e r m s} \mathbf{- 1}$ and $\tau=1-T / T_{c}$

Thermal Conductivity $\lambda$ (Gas at low pressures; $p<6$ bar)

- DEFAULT: TransportPolynomial: TransportPolynomial-ThermCondGas $\lambda=\sum \mathrm{a}_{\mathrm{i}} \boldsymbol{T}^{\mathrm{i}}$, where the summation is from $\boldsymbol{i}=\mathbf{0}$ to $\boldsymbol{n T e r m s}-\boldsymbol{1}$

- Alternative 1: Yaws.PolynomialExpansion Yaws.PolynomialExpansionThermCondGas $\lambda=\sum \mathrm{a}_{\mathrm{i}} \cdot \boldsymbol{T}^{\mathrm{i}}$, where the summation is from $\boldsymbol{i}=\mathbf{0}$ to $\boldsymbol{n T e r m \boldsymbol { s }} \mathbf{- 1}$

- Alternative 2: PPDS 3 PPDS3-ThermCondGas $\lambda=T_{r}^{0.5}\left(\sum \mathrm{a}_{\mathrm{i}} / T_{r}^{\mathrm{j}}\right)^{-1}$, where the summation is from $\boldsymbol{i}=\mathbf{1}$ to $\mathbf{3}$, and $T_{r}=T / T_{c}$, where $T_{c}$ is the critical temperature. 